\title{
Experimental Demonstration of Pulse Shaping and Shortening by Spatial Filtering of an Induced-Phase-Modulated Probe Wave
}

\author{
A. Dreischuh, I. Buchvarov, E. Eugenieva, A. Iliev, and S. Dinev
}

\begin{abstract}
An asymmetrically induced phase modulation resulting in an intensity-dependent spatial beam deflection and a subsequent spatial filtering have been used for all-optical pulse shaping and shortening. A compression factor of eight and controllable symmetrical super-Gaussian pulses and pulse trains have been observed in a good agreement with previous analyses in a pump-probe beam configuration.
\end{abstract}

Index Terms - Laser beam distortion, nonlinear optics, optical pulse shaping.

\section{INTRODUCTION}

$\mathbf{L}$ IGHT pulses of special temporal shapes are needed in many areas of modern optics [1]-[3], e.g., timeresolved spectroscopy, nonlinear optics in fibers, optical communications, and data processing. A variety of pulse-shaping techniques, based on different physical phenomena, have been developed in recent years. One of these approaches allows programmable pulse shaping of ultrashort pulses by a spatial spreading of the spectral components of the light pulse and modifying their amplitude and/or phase in order to produce a spectrum that corresponds to the desired pulse shape [4]-[8]. Alternative approaches include spectral beam deflection [9], temporal pulse shaping in a grating pair compressor [10], cross-phase-modulation-induced compression [11], high-speed electrooptic deflection [12], and light-induced deflection and spatial filtering of an optical beam/pulse [13].

Light-bending effects in Kerr media have been studied extensively during the last few years [13]-[20] because of their potential applications for all-optical control of light beams/pulses. These phenomena originate in the refractive index change along/across the nonlinear medium due to selfor cross-phase modulation. Spatial phase modulation of the light wave at the entrance face of the nonlinear sample or acquired during the propagation of the light beam/pulse along the nonlinear medium plays an important role for the observation of both self- and induced deflection [17], [18]. At certain conditions, the spatial phase modulation can affect considerably the temporal behavior of the light pulse [17], [19].

Self-bending of a light beam due to Kerr effect was first proposed by Kaplan [22] and verified experimentally by Brodin

Manuscript received December 18, 1995; revised August 21, 1996. This work was supported by the National Science Foundation, Bulgaria under Contract F-209.

The authors are with Sofia University, Department of Quantum Electronics, BG-1164, Sofia, Bulgaria.

Publisher Item Identifier S 0018-9197(97)01567-4.
[23]. Self-deflection (or self-bending) of an asymmetrical CW laser beam was observed by Swartzlander et al. [24]. Induced deflection and focusing in a self-defocusing medium have been reported [20], [21]. Self-deflection in $\mathrm{CS}_{2}$ and subsequent spatial filtering through a slit placed in the far-field region have been used to shorten picosecond laser pulses [14]. In a recent paper, dynamical properties of light-bending effects have been analyzed and optimized [15].

In a previous paper, we have proposed a new technique for shaping and shortening of optical pulses, based on their induced deflection and slicing [13].

The aim of this work is to provide experimental evidences on the possibility to change controllably the shape and, simultaneously, to shorten (in time) probe laser pulses. The comparative numerical results presented are in good agreement with the experimental data.

\section{PhysicalMechanisms}

When two intense laser beams copropagate in a thirdorder nonlinear medium, they are incoherently coupled by the cross-phase modulation. In a pump-probe configuration, the refractive-index correction at the probe wavelength/polarization is a result of the pump-induced phase modulation (IPM). At a nonzero initial angular deviation and/or off-axial separation $x_{0}$ between the beams, the asymmetrical refractive-index correction across the probe beam should lead to its far-field spatial deflection. This type of an optically induced probe-beam deflection should be intensity dependent. As a result, if temporally synchronised pulses are considered instead of beams, the leading and trailing wings of the probe pulse should be less deflected as compared to the central part of the pulse. In view of the above assumptions, a simple spatial filtering of the deflected probe wave (by a diaphragm, slit, etc.) could be expected to result in the generation of optical pulses of special shapes and reduced duration. From a certain point of view, the physical picture described is entirely based on the spatio-temporal coupling of the two waves involved and the pulse shaping and shortening is due to pulse slicing.

\section{EXPERIMENTALSCHEME}

The experimental scheme used is sketched on Fig. 1. The output of a $Q$-switched Nd:YAG laser $\left(\tau_{\text {imp }}=22 \mathrm{~ns}\right)$ is frequency-doubled in a nonlinear crystal (SHG) in a firsttype nonlinear process. Both first- and second-harmonic beams 


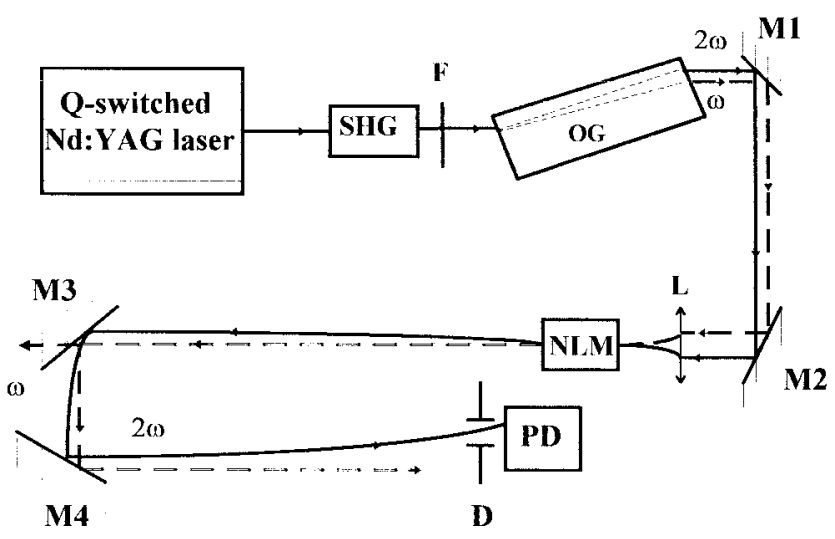

Fig. 1. Experimental scheme. SHG-nonlinear crystal for second harmonic generation; F-filter; OG-bulk piece of optical glass; M1, M2, M4-mirrors; M3-dichroic mirror; D—diaphragm; PIN-photodiode; L-lens; NLM—nonlinear medium.

enter a tilted bulk piece of optical glass (OG). Due to the wavelength dispersion, the pump and the probe beams appear with an off-axial separation $x_{0}$ of approximately $800 \mu \mathrm{m}(0.6$ times the pump beam radius). Both beams are focused with a spherical lens $(f=50 \mathrm{~cm})$ in a 5 -cm-long quivette filled with nitrobenzene. This nonlinear medium (NLM) posses an orientational Kerr nonlinearity of a picosecond response time and a polarization-dependent sign of its orientational nonlinear susceptibility. The asymmetrically induced phase-modulated (AIPM) probe beam becomes spatially deflected in the farfield. The temporally integrated probe beam distribution is recorded with a CCD camera and a frame-grabber. The time evolution of the deflected probe beam transmitted trough the far-field diaphragm D is recorded with a PIN-photodiode (PD) (response time of approximately $800 \mathrm{ps}$ ) and a $250-\mathrm{MHz}$ storage oscilloscope. In reality, the spatial filtering of the deflected probe beam/pulse is a result of the small aperture of the photodiode $\left(\sim 1 \mathrm{~mm}^{2}\right)$. In order to scan across the probe beam cross section, the diode is mounted on a translation stage.

\section{NUMERICALMODEL}

The $(2+1)$ dimensional model developed is formulated under the assumption of a negligible linear spatio-temporal evolution of the beams inside the NLM. Since nanosecond pulses are used, the group-velocity mismatch was also neglected. These assumptions allow one to treat the time coordinate as a parameter. Therefore, within the thin NLM, the asymmetrical IPM should be accounted for in the phase portrait of the probe wave only. According to the above assumptions, at the entrance face of the NLM, the pump and probe wave are described by

$$
\begin{aligned}
A_{p}(x, y, t ; z=0)= & A_{p 0} \exp \left\{-\left[\left(x-x_{0}\right)^{2}+y^{2}\right] / a_{p}^{2}\right\} \\
& \cdot \exp \left\{-t^{2}\right\}
\end{aligned}
$$

and

$$
A_{s}(x, y, t, z=0)=A_{s 0} \exp \left\{-\left[x^{2}+y^{2}\right] / a_{s}^{2}\right\} \exp \left\{-t^{2}\right\}
$$

respectively, where $a_{s}$ and $a_{p}$ are the corresponding probe and pump beam radii, and $x_{0}$ is their initial mutual off-axis offset.
Within the NLM of a length $l_{\mathrm{NL}}$, a nonlinear phase

$$
\Phi_{s}^{\mathrm{NL}}(x, y, t)=k_{s} l_{\mathrm{NL}} n_{2}^{\mathrm{IPM}}\left|A_{p}(x, y, t)\right|^{2}
$$

is induced on the probe beam/pulse. In (1)-(2), the local time coordinate denoted with $t$ is normalized to the root mean square of the pump and probe pulse durations. The use of normalized units [see also (4)] makes the numerical results obtained more general (provided the above assumptions are fulfilled), underlining that all the shaped probe pulses obtained in this way are simultaneously shortened as compared to the incoming ones. One hundred time-slices are calculated within the pulse full-width and a total of 250 are considered in calculating the filtered pulse-shapes and the time-integrated energy-density distributions of the probe wave [see Fig. 4(a)]. The Fresnel-Kirchoff diffraction integral describing the transformation of the probe field distribution from the exit of the NLM

$$
A_{s}\left(x, y, t, z=l_{\mathrm{NL}}\right)=A_{s}(x, y, t, z=0) e^{i \Phi_{s}^{\mathrm{NL}}(x, y, t)}
$$

to the far-field (i.e., to the aperture plane) could be simplified [17], [25] to a Fourier-transformation integral. At each local time within the probe pulse, the 2-D Fourier transformations (in space) are expanded over $256 \times 256$ grid points. The stability of the results was proved by doubling simultaneously the number of the time-slices and the grid-mesh discretization.

\section{EXPERIMENTAL ANDNUMERICALRESULTS: COMPARATIVEANALYSIS}

For a better understanding of the results obtained, it is worth noting that the time-integrated far-field energy density distributions recorded by the CCD-camera could differ significantly from the momentary intensity distribution of the deflected probe wave. In media with instantaneous (with respect to the pulse durations) nonlinear response, this behavior should be attributed to the intensity-dependent (self-) induced deflection.

Fig. 2 shows the typical temporal profile of the probe pulses at the second harmonic of the Nd:YAG laser used $\left(\lambda_{s}=532\right.$ $\mathrm{nm})$. The pulse-width is $\tau_{s}=22 \mathrm{~ns}$ FWHM. Along with the well-expressed stability of the shape, the relatively low $(\sim 4 \%)$ pulse-to-pulse energy fluctuation is to be mentioned. Fig. 3 presents two typical far-field time-integrated images of the probe beam. The first one [Fig. 3(a)] shows the probe beam transverse profile in the linear regime of propagation. This regime corresponds to a pump wave being blocked out and to a filter set F (see Fig. 1) suppressing the eventual selfaction of the probe within the NLM. The latter is ensured in all measurements. Fig. 3(b) shows the typical far-field timeintegrated energy density distribution of the probe wave. The dashed line indicates the interaction axis $[x$; see (1)]. Weak modulation in the wing of the deflected probe beam could be observed. The enhanced beam cross section is a result of the induced probe beam defocusing and deflection at $n_{2}^{\mathrm{IPM}}\left(\lambda_{s}\right)<$ 0 . At crossed pump and probe polarizations, a negative sign of the nonlinear refractive-index coefficient should be expected in an orientational Kerr nonlinear medium. In order to avoid the CCD-camera saturation, Fig. 3(a) is recorded with a neutral density filter. 


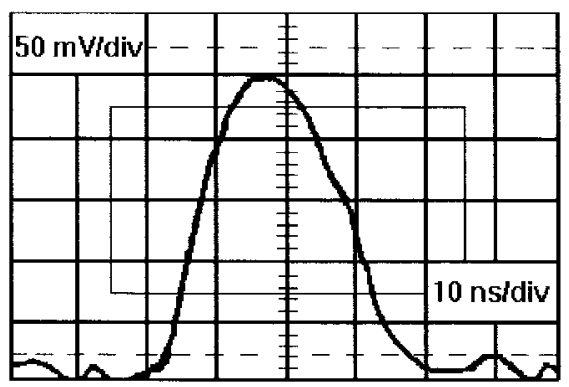

Fig. 2. Typical shape of the probe pulses used in the experiment $\left(\lambda_{s}=532\right.$ $\mathrm{nm})$.

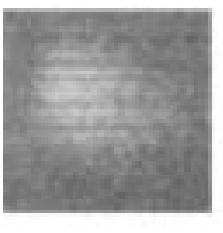

(a)

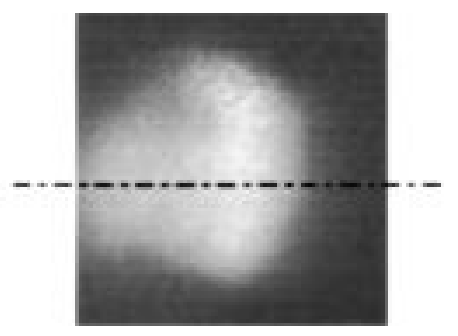

(b)
Fig. 3. Time-integrated grayscale images of (a) the undistorted and (b) the deflected probe beam.

In Fig. 4(a), we plot both the time-integrated (solid line) and instantaneous (at $t=0$ ) far-field probe beam profiles obtained by the numerical model formulated in Section IV. Both slices are extracted along the interaction axis [see Fig. 3(b)] i.e., at $y=0$. The initial off-axis distance $x_{0}=0.6 a_{p}$ and the probeto-pump beam radii ratio $a_{s} / a_{p}=0.7$ assumed in the model closely correspond to the measured values $\left(a_{p}=1.3 \mathrm{~mm}\right.$, $a_{s}=0.9 \mathrm{~mm}, x_{0}=0.8 \mathrm{~mm}$ ) at the focusing lens. In order to describe adequately the strength of the AIPM, we used as a fitting parameter the quantity

$$
N=\Phi_{s}^{\mathrm{NL}}\left(x=x_{0}, y=0, z=l_{\mathrm{NL}}, t=0\right) /(2 \pi) .
$$

The goal of this procedure is to achieve a close similarity between the time-integrated far-field probe beam profile from the numerical simulations [Fig. 4(a), solid curve) and the one from the experiment [Fig. 4(b)]. At the typical pulse energy of $7.5 \mathrm{~mJ}$ and $0.260-\mathrm{mm}$ pump beam radius at the entrance face of the NLM, the pump intensity was estimated to be $I_{p}(z=0)=$ $150 \mathrm{MW} / \mathrm{cm}^{2}$, yielding $|N|=4.9$. This value is estimated by using the known value of the nonlinear refractive index $n_{2}^{\text {IPM }}=5.53 \cdot 10^{-14} \mathrm{~cm}^{2} / \mathrm{W}$ [26] for nitrobenzene, keeping in mind that at crossed polarizations the sign of the nonlinear refractive-index correction for the probe wave is negative [27]. This value could be slightly overestimated because of the electronic contribution to $n_{2}^{\text {IPM }}$ [27] and seems to agree well with the value $N=-4.5$ assumed in the rest of this paper. Several points are denoted identically on Fig. 4(a)-(b), indicating the specific positions of the aperture/photodiode in the simulation and the experiment, respectively. At these specific spatial positions, the experimental probe pulse shaping and shortening will be compared with the numerical simulation. In order to clarify the possibility to form trains of probe pulses, the dashed curve on Fig. 4(a) presents the transverse probe

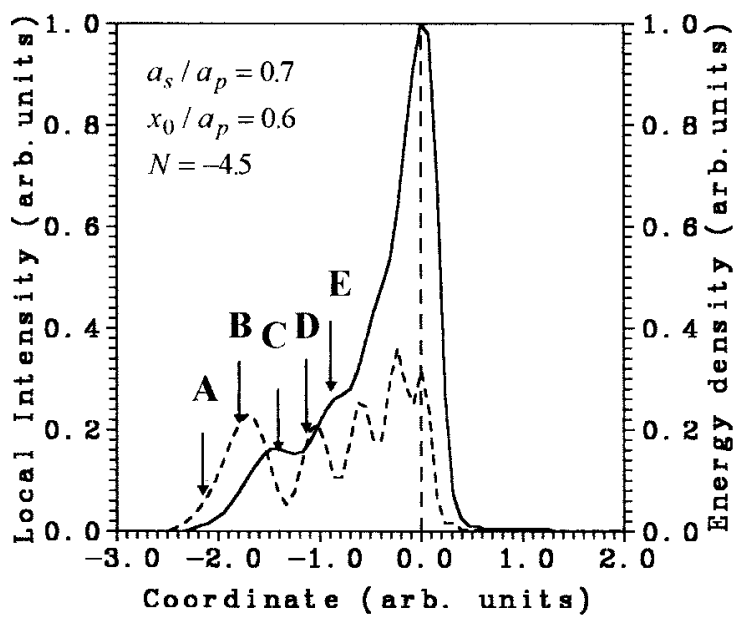

(a)

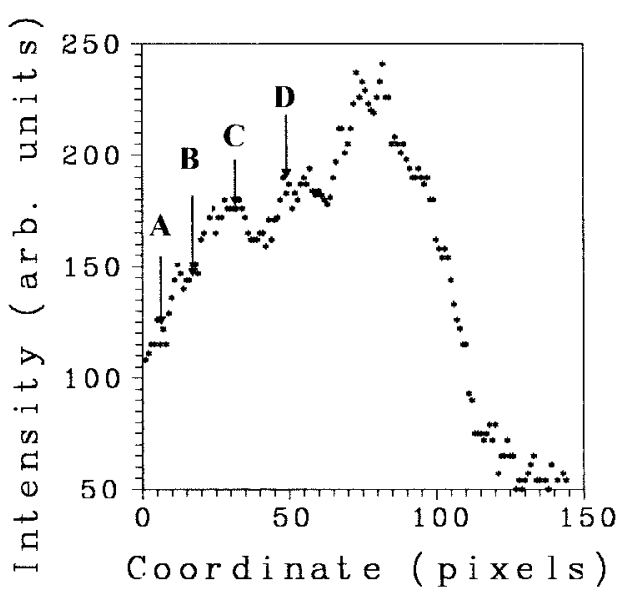

(b)

Fig. 4. (a) Results obtained from the numerical simulations (solid line-temporally-averaged probe beam profile; dashed line-probe beam shape at a local time $t=0$ ). The vertical dashed line indicates the probe beam axis in the linear regime of propagation. (b) Time-integrated probe beam cross section along the interaction axis [extracted from Fig. 3(a)].

beam intensity distribution along the interaction axis in the common peak of the pulse (i.e., at $t=0$ ).

At a certain pump peak intensity (i.e., at a fixed value of $N$ ), optimal probe pulse shortening can be achieved by filtering the most deflected part of the probe beam through the aperture [see points A on Fig. 4(a)-(b)]. The experimental result obtained is presented on Fig. 5(a) and the corresponding 2-D numerical one-on Fig. 5(b). The duration of the short pulse obtained seems less than 3 ns and could be determined more accurately by using an oscilloscope of broader bandwidth and a faster photodiode. The numerical data [Fig. 5(b)] indicate that the shape of this maximum shortened pulse should be Gaussian if Gaussian input pulses are used. The shortening factor in the experiment is greater than 7, two times higher than the one obtained from our 2-D model. This deviation could be explained in the following way. When axially offset beams are focused by a spherical lens, aberrations and angular beam off-set should be present at the entrance face of the nonlinear medium (see Fig. 1). As shown in [13], small angular deviations may appear to be better suited for achieving 


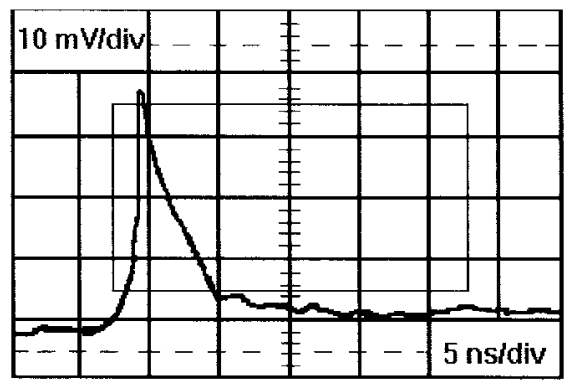

(a)

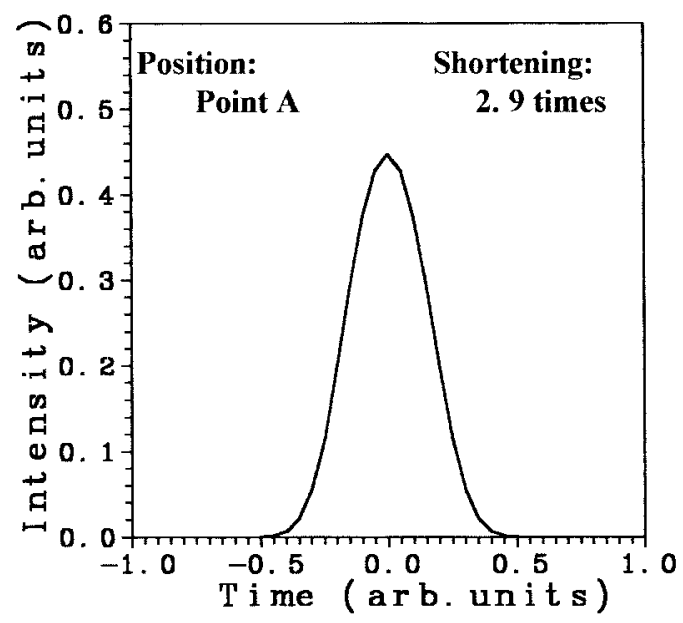

(b)

Fig. 5. (a) Optimal pulse shortening [experimental result; detector position point A on Fig. 4(b)]. The corresponding numerical results shows a temporal symmetry of the transmitted pulse [see point A on Fig. 4(a)].

larger probe-beam deflection and shortening. It is interesting to note that our $(1+1)$ dimensional simulations under the same conditions yield a shortening factor of 7.5 under the same conditions. This fact, however, is to be explained by the $1-\mathrm{D}$ probe-beam energy redistribution.

By placing the aperture slightly closer to the linear probebeam axis [in the vicinity of points B on Fig. 4(a)-(b)], superGaussian signal pulses are generated experimentally [Fig. 6(a)] in an agreement with the numerical simulations [Fig. 6(b)]. The power of the super-Gaussian, (i.e., the slant of the pulse leading and trailing edge) is difficult to estimate because the rise/fall time of these fronts is limited by the bandwidth of the data acquisition equipment used. Nevertheless, the flat-toped peak of the signal generated [Fig. 6(a)] is indicative for its super-Gaussian profile.

Let us consider in more detail the specific spatial farfield position denoted by point $\mathrm{C}$ on Fig. 4(a)-(b). As seen, this position corresponds to the most deflected peak in the temporally integrated probe beam spatial distribution. The central part of the probe pulse (at $t=0$ ), however, is more gradually deflected [Fig. 4(a), the dashed line near point B). Because of the decreasing local intensity in the pulse wings, certain local times, at which the deflected probe wave passes through the diaphragm, should exist. As a consequence, a pair of identical signal pulses with durations shorter than those of the probe ones are observed in the experiment [Fig. 7(a)] and and in the simulation [Fig. 7(b)]. The relatively low

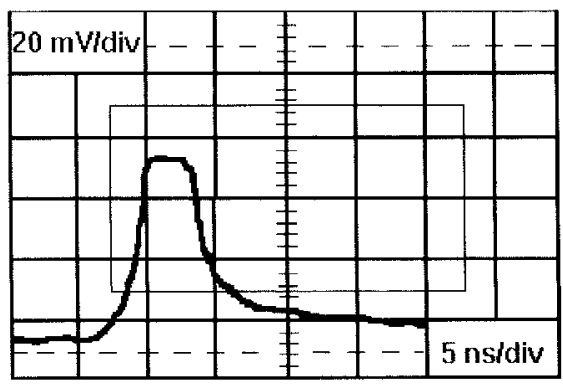

(a)

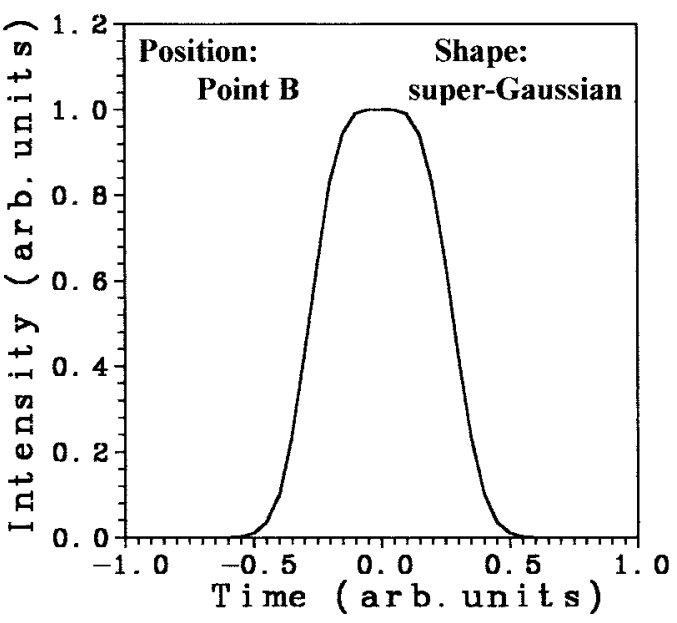

(b)

Fig. 6. (a) Experimental and (b) numerical results confirming the possibility to generate super-Gaussian signal pulses. [detector position-points B on Figs. 4(a) and (b)]

modulation depth of the experimental pulse shape could be attributed, at least partially, to the relatively slow response of the equipment used. The pair of pulses simulated numerically is of a better contrast, but the enhancement of the pulse discretization should lead to even more representative results.

At a diaphragm/detector position indicated by point $\mathrm{D}$ [see Fig. 4(a)-(b)], transmission of a sequence of three pulses is observed both experimentally [Fig. 8(a)] and numerically [Fig. 8(b)]. Each signal pulse from this sequence is shorter than the spatially deflected probe pulse. Moreover, it could be expected that a $M$-pulse sequence, consisting of single signal pulses of durations $\tau_{\text {signal }}=\tau_{s} /(2|N|-1)$, could be formed at an effective nonlinearity $|N|=M / 2$. In the particular case of $N=-4.5$ and a diaphragm located at point D [Fig. 4(a)-(b)], each sliced pulse should be eight times shorter than the incoming signal pulse. The experimental value [see Fig. 8(a)] seems to be very close to this simple estimation. Unfortunately, once again, the experimental curve seems limited by the temporal resolution of the data-acquisition system. The difference of the peak-to-peak contrast within the experimental [Fig. 8(a)] and theoretical [Fig. 8(b)] pulse train seems to be a manifestation of this limitation.

The possibility to generate a multiple-pulse sequence within the temporal profile of a single probe pulse is proved numerically by locating the diaphragm at position E on Fig. 4(a), even closer to the linear probe-beam axis. This particular position does not correspond to an extremum in the time-integrated 


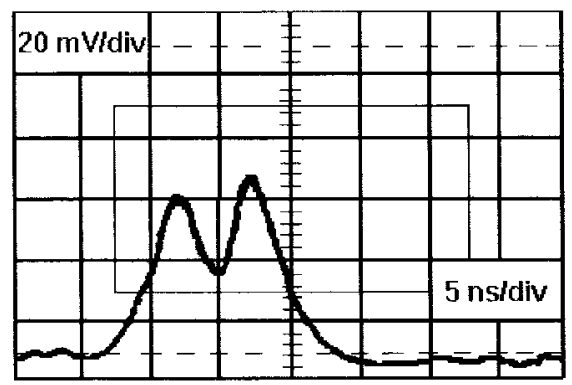

(a)

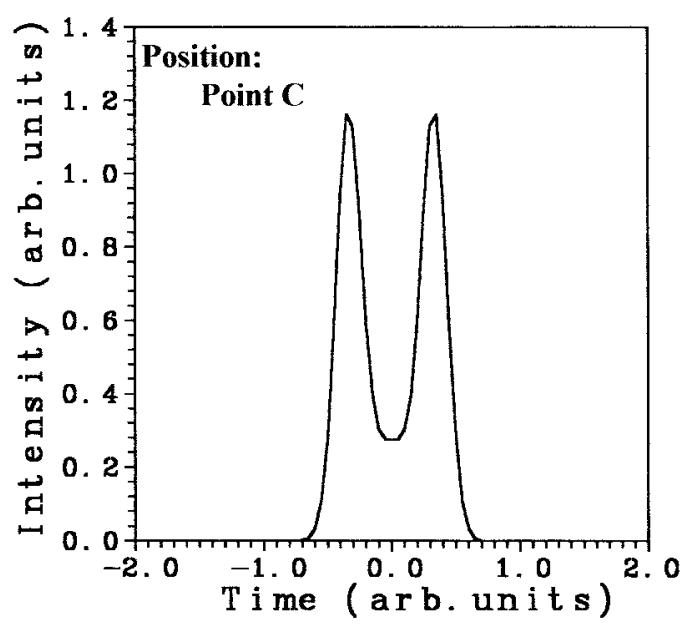

(b)

Fig. 7. (a) Experimental (b) and numerical results confirming the possibility to generate pairs of identical signal pulses. [Detector position-points $\mathrm{C}$ on Fig. 4(a) and (b).]

slice along the interaction axis (solid curve), but it corresponds to an intensity minimum at a local time $t=0$, (i.e., at the peak of the pulse, Fig. 4(a), dashed curve). A diaphragm positioned in this way should transmit four successive signal pulses (Fig. 9). A corresponding experimental result could not be recorded, most probably due to the relatively slow response time of the data-acquisition system. Sequences consisting of up to seven pulses are observed in the numerical simulation at $N=-4.5$ with the same discretization (see Section IV).

In our view, there is an analogy between the spatiotemporal interaction scheme analyzed and some time-domain induced/cross-phase modulation phenomena in optical fibers. This analogy could provide an additional physical insight to why multiple pulses in sequences arised. It is shown [28], [29] that the IPM at a nonzero group-velocity mismatch and walk-off (along an optical fiber) can lead to an asymmetric pulse spectral broadening. The AIPM and far-field probe beam deflection considered in this work could be regarded as a spatial analog of the induced frequency-shift described in these works [28], [29]. At this stage, the only difference consists in the abrupt change of the regime of propagation from nonlinear to a purely linear one at the exit face of the NLM in the present case. In [21], the oscillations in the wing of the induced-deflected waves are considered as resulting from a process, which is a spatial analog of the optical wave-breaking phenomenon extensively studied in optical fibers. It should be noted that the positions of the deflected peaks within the probe

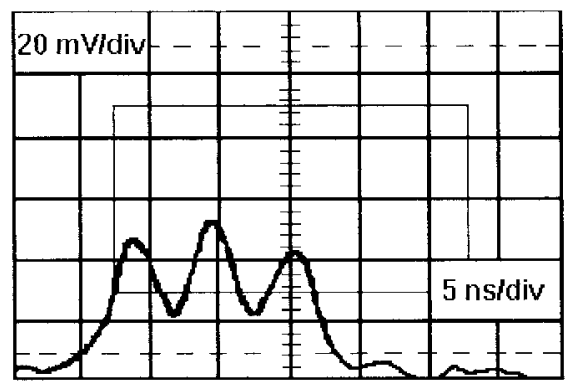

(a)

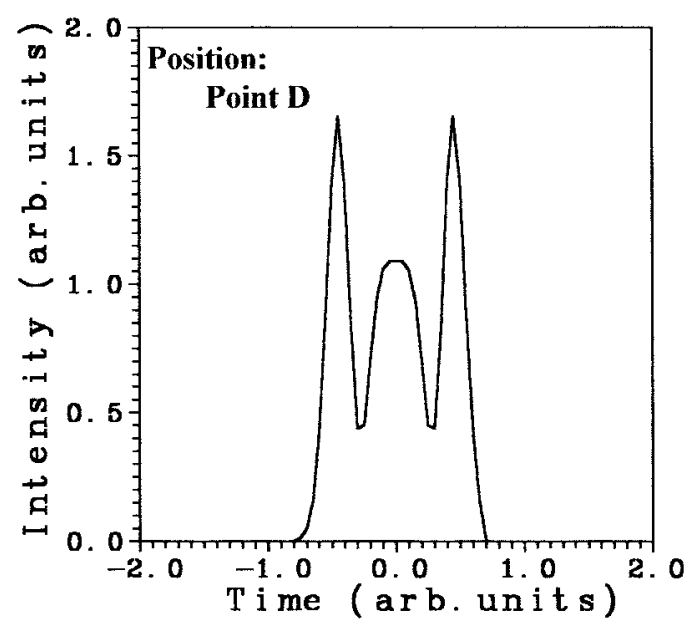

(b)

Fig. 8. Sequence of three pulses (a) experimentally and (b) numerically obtained with a diaphragm/detector positioned at point D on Fig. 4(b) and (a), respectively.

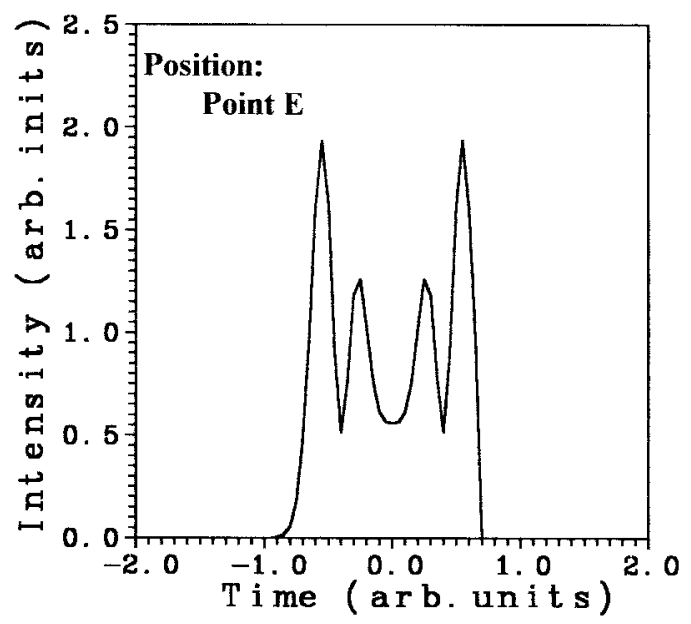

Fig. 9. Four-pulse sequence obtained at a detector position located closer to the linear probe beam axis [see point E in Fig. 4(a)].

beam and the number of these peaks does depend on the local pump-beam intensity (i.e., on the local time within the pump pulse; see [13, Fig. 3]). At certain local times within the pulse envelope, the aperture transmits parts of the deflected probe wave only if a peak from its ringing wing passes across. As a consequence, the number of the pulses being sliced by the spatial filter could not exceed two times the number of the peaks in the wing of the deflected probe wave at its temporal peak (at $t=0$ ). 
In all simulations, the peak intensities of the central pulses within the sequence are found to be lower than those of the leading and trailing pulses of the series. Intuitively, this behavior could be explained by the following. At a diaphragm/detector located near the probe beam axis, the leading and trailing pulses of the sequence are formed from the spatially dispersed leading and trailing fronts of the pulse. The local (in time) intensity of the pulse wing is relatively small as compared to the peak intensity and the corresponding 2-D local energy density redistribution is relatively weak. The central high-intensity part of the pulse becomes stronger phase modulated, most gradually deflected, and the 2-D energy redistribution is the most pronounced one. As a result, the spatially filtered pulses should be expected to be of reduced intensity. It should be noted that all results presented and qualitative explanations refer to the case of synchronized pump and probe pulses with zero initial mutual delay.

The energy efficiencies are calculated as follows. In the linear regime of propagation, i.e., at $N=0$, no induced deflection occurs. The diaphragm is placed on-axially with respect to the undeflected probe beam and the total energy $W_{0}$ of the transmitted pulse is calculated. The total energy of the pulses and pulse trains transmitted at different positions of the diaphragm are normalized to $W_{0}$. The energy efficiencies are $0.34 \%, 1.1 \%, 1.35 \%, 2.4 \%$, and $2.9 \%$ for the maximum shortened pulse (Fig. 5), super-Gaussian pulse (Fig. 6), and the sequences of two (Fig. 7), three (Fig. 8), and four (Fig. 9) pulses, respectively. The values corresponding to the trains of pulses refer to the energy of the whole train. These energy efficiencies are comparable to the values in the 1-D case without an initial angular deviation between the pump and probe beams [13].

Since the pulse shaping and shortening effects result from a spatio-temporal slicing of the deflected probe wave, the spatial filter (aperture) influences its beam quality. In Table I, we summarize our results on the near- and far-field beam-quality factors [30] for the pulses and pulse sequences discussed. The positions of the planes are denoted as near- and farfield with respect to the distances behind the aperture. The numerical data presented are obtained at an aperture of a radius equal to the pump-beam radius at the entrance of the NLM. The first numbers in Table I refer to the beam-quality factors along the interaction axis, whereas the numbers in brackets - to the perpendicular axis [y, see (1a)]. In the near field behind the aperture, the quality of the time-integrated beam profile is found to be better than that obtained for the central part of the filtered probe pulse (at $t=0$ ). This is to be attributed to the time-integration of the spatially deflected probe wave within the aperture. The far-field beam-quality factors were found to be typically equal to 1.04 independently of the evaluation direction-to-interaction axis orientation for both time-integrated and peak-intensity distributions.

\section{CONCLUSION}

In this proof-of-principle experiment described, we demonstrate a simultaneous shaping and shortening of nanosecond probe laser pulses. Since the asymmetric induced phase modulation is an incoherent effect, shaping and shortening of pulses
TABLE I

Near- And Far-field Beam-Quality Factors of the Filtered Probe Waves at Different Aperture Positions Yielding MaXimum Shortened Pulse (MSP), Super-gaussian (SG) Pulse, Pair of Pulses (PP), AND Three- AND Four-Pulse Sequences (TPS and FPS, ResPectively)

\begin{tabular}{lcccccc}
\hline \multirow{2}{*}{ Plane } & \multicolumn{5}{c}{ Beam quality } \\
\cline { 2 - 7 } & & MSP & SG & PP & TPS & FPS \\
\hline \multirow{2}{*}{$\begin{array}{l}\text { Near } \\
\text { field }\end{array}$} & integrated & $1.24(1.70)$ & $1.42(1.70)$ & $1.72(1.70)$ & $1.53(1.70)$ & $1.62(1.75)$ \\
\cline { 2 - 7 } & at $\mathrm{t}=0$ & $1.30(1.71)$ & $1.60(1.71)$ & $1.84(1.71)$ & $1.49(1.71)$ & $1.94(1.71)$ \\
\hline \multirow{2}{*}{$\begin{array}{l}\text { Far } \\
\text { field }\end{array}$} & integrated & $1.04(1.04)$ & $1.04(1.04)$ & $1.03(1.04)$ & $1.04(1.04)$ & $1.03(1.04)$ \\
\cline { 2 - 7 } & at -0 & $1.04(1.03)$ & $1.04(1.04)$ & $1.05(1.04)$ & $1.04(1.04)$ & $1.05(1.04)$
\end{tabular}

The numbers in brackets refer to the beam-quality factors in a direction, perpendicular to the interaction axis denoted in Fig. 3.

in a wide spectral range should be possible if nonresonant NLM are used. It will be interesting to estimate the limitations of this technique when (sub)picosecond initial pulses are used. Our preceding numerical data are indicative for the possibility to generate, for instance, triangular-like signal pulses at nonzero initial pump-to-probe delay. An advantage of the scheme analyzed is that no initial ultrashort pulses are needed.

\section{REFERENCES}

[1] A. M. Weiner, Y. Silberberg, H. Fouckard, D. Leaird, M. A. Saifi, M. J. Andrejco, and P. W. Smith, "Use of femtosecond square pulses to avoid pulse breakup in all-optical switching," IEEE J. Quantum Electron., vol. 25 , pp. 2648-2655, 1989, and references therein.

[2] G. Aureli, S. Betfi, G. de Marchis, E. Iannone, and A. Mecozzi, "Theoretical analysis of optical pulse position modulation communication systems using semiconductor lasers," Opt. Quantum Electron., vol. 23, pp. 1077-1089, 1991.

[3] A. M. Weiner, D. E. Leaird, D. H. Reitze, and E. G. Paek, "Femtosecond spectral holgraphy," IEEE J. Quantum Electron., vol. 28, pp. 2251-2261, 1992.

[4] C. Froehly, B. Colombeau, and M. Vampouille, "Shaping and analysis of picosecond light pulses," in Progress in Optics XX, E. Wolf, Ed. Amsterdam, The Netherlands: North-Holland, 1983, pp. 65-153.

[5] A. M. Weiner, J. P. Heritage, and E. M. Kirschner, "High-resolution femtosecond pulse shaping," J. Opt. Soc. Amer. B, vol. 5, pp. 1563-1572, 1988.

[6] D. H. Reitze, A. M. Weiner, and D. E. Leaird, "Shaping of wide bandwidth 20 femtosecond optical pulses," Appl. Phys. Lett., vol. 61, pp. 1260-1262, 1992.

[7] A. M. Weiner, D. E. Leaird, J. S. Patel, and J. R. Wullert, "Programmable femtosecond pulse shaping by use of multielement liquid crystal phase modulator," Opt. Lett., vol. 15, pp. 326-328, 1990.

[8] M. M. Wefers and K. A. Nelson, "Analysis of programmable ultrashort waveform generation using liquid-crystal spatial light modulators," $J$. Opt. Soc. Amer. B, vol. 12, pp. 1343-1362, 1995.

[9] S. Skupsky, T. J. Kessler, S. A. Letzring, and Y.-H. Chuang, "Laserbeam pulse shaping using spectral beam deflection," J. Appl. Phys., vol. 73, pp. 2678-2685, 1993.

[10] A. Penzkofer, "Theoretical analysis of pulse shaping of self-phase modulated pulses in a grating pair compressor," Opt. Quantum Electron., vol. 23, pp. 685-702, 1991, and references therein.

[11] G. P. Agrawal, P. L. Baldeck, and R. R. Alfano, "Temporal and spectral effects of XPM on copropagating ultrashort pulses in optical fibers," Phys. Rev. A, vol. 40, pp. 5063-5072, 1989.

[12] B. Lee, T. Kobayashi, A. Marimoto, and T. Sueta, "High-speed electrooptic deflector and its applications to picosecond pulse generation," IEEE J. Quantum Electron., vol. QE-28, pp. 1739-1744, 1992.

[13] A. Dreischuh, E. Eugenieva, and S. Dinev, "Pulse shaping and shortening by spatial filtering of an induced-phase-modulated probe wave," IEEE J. Quantum Electron., vol. 30, pp. 1656-1661, 1994. 
[14] A. Barthelemy, C. Froehly, S. Maneuf, and F. Reynaud, "Experimental observation of beams' self-deflection appearing with two-dimensional spatial soliton propagation in bulk Kerr material," Opt. Lett., vol. 17, pp. 844-846, 1992.

[15] H. Ma and C. B. De Araujo, "Time shortening of laser pulses as an application of dynamical light bending effects," Opt. Comm., vol. 110, pp. 615-619, 1994

[16] H. Ma and S. B. de Araujo, "Light-controlled beam deflector in semiconductor dopped glasses," Appl. Phys. Lett., vol. 63, pp. 3553-3555, 1993.

[17] N. Goutev, A. Dreischuh, S. Balushev, and S. Dinev, "2D asymmetric induced phase modulation: Spatial and spatio-temporal aspects," IEEE J. Quantum Electron., vol. 31, pp. 2114-2119, 1995.

[18] X. D. Cao, D. D. Meyerhopher, G. P. Agrawal, "Optimization of optical beam steering in nonlinear Kerr media by spatial phase modulation," $J$. Opt. Soc. Amer. B, vol. 11, pp. 2224-2231, 1994.

[19] A. T. Ryan and G. P. Agrawal, "Pulse compression and spatial phase modulation in normally dispersive nonlinear Kerr media," Opt. Lett., vol. 20, pp. 306-308, 1995

[20] G. P. Agrawal, "Induced focusing of optical beams in self-defocusing nonlinear media," Phys. Rev. A, vol. 64, pp. 2487-2490, 1990.

[21] A. J. Stentz, M. Kauranen, J. Maki, G. P. Agrawal, and R. W. Boyd, "Induced focusing and spatial wavebreaking from XPM in a selfdefocusing medium," Opt. Lett., vol. 17, pp. 19-21, 1992.

[22] A. E. Kaplan, "Bending of trajectories of asymmetrical laser beams in nonlinear media," JETP Lett., vol. 9, pp. 3-7, 1969, (vol. 9, pp. 58-62, 1969, in Russian).

[23] M. S. Brodin and A. M. Kamuz, "Observation of self-bending of a nonuniform intense laser beam in an $\mathrm{NaCl}$ crystal," JETP Lett., vol. 9, pp. 351-354, 1969 (vol. 9, pp. 577-580, 1969, in Russian).

[24] G. A. Swartzlander, Jr., H. Yin, and A. E. Kaplan, "Continuous-wave self-deflection effect in sodium vapor," J. Opt. Soc. Amer. B, vol. 6, pp. 1317-1325, 1989.

[25] S. Huges, J. M. Burzler, G. Spruce, and B. S. Wherrett, "FFT techniques for efficient simulation of Z-scan measurements," J. Opt. Soc. Amer. B, vol. 12, pp. 1888-1893, 1995.

[26] M. J. Weber, CRC Handbook of Laser Science and Technology. Bocca Raton, FL: CRC Press, 1986, part I, vol. III, p. 269.

[27] M. Sheik-Bahae, J. Wang, R. DeSalvo, and E. W. Van Stryland, "Measurement of nondegenerate nonlinearities using a two-color Zscan," Opt. Lett., vol. 17, pp. 258-260, 1992.
[28] P. L. Baldeck, R. R. Alfano, and G. P. Agrawal, "Induced-frequency shift of copropagating pulses," Appl. Phys. Lett., vol. 52, pp. 1939-1941, 1988.

[29] R. R. Alfano, P. L. Baldeck, P. P. Ho, and G. P. Agrawal, "Cross-phase modulation and induced focusing due to optical nonlinearities in optical fibers and bulk materials," J. Opt. Soc. Amer., vol. B6, pp. 824-829, 1989.

[30] A. E. Siegman and S. W. Townsend, "Output beam propagation and beam quality from a multimode stable-cavity laser," IEEE J. Quantum Electron., vol. 29, pp. 1212-1217, 1993.

A. Dreischuh, photograph and biography not available at the time of publication.

I. Buchvarov, photograph and biography not available at the time of publication.

E. Eugenieva, photograph and biography not available at the time of publication.

A. Iliev, photograph and biography not available at the time of publication.

S. Dinev, photograph and biography not available at the time of publication. 